\title{
Two-loop corrections to Starobinsky-Higgs inflation
}

\author{
D. M. Ghilencea* \\ Theoretical Physics Department, National Institute of Physics and Nuclear Engineering (IFIN), \\ Bucharest 077125, Romania
}

(Received 4 August 2018; published 26 November 2018)

\begin{abstract}
Higgs inflation and $R^{2}$ inflation (Starobinsky model) are two limits of the same quantum model, hereafter called Starobinsky-Higgs. We analyze the two-loop action of the Higgs-like scalar $\phi$ in the presence of (1) nonminimal coupling $(\xi)$ and (2) quadratic curvature terms. The latter are generated at the quantum level with $\phi$-dependent couplings $(\tilde{\alpha})$ even if their tree-level couplings $(\alpha)$ are tuned to zero. Therefore, the potential always depends on both Higgs field $\phi$ and scalaron $\rho$; hence, multifield inflation is a quantum consequence. The effects of the quantum (one- and two-loop) corrections on the potential $\hat{W}(\phi, \rho)$ and on the spectral index are discussed, showing that the Starobinsky-Higgs model is, in general, stable in their presence. Two special cases are also considered: first, for a large $\xi$ in the quantum action, one can integrate $\phi$ and generate a "refined" Starobinsky model which contains additional terms $\xi^{2} R^{2} \ln ^{p}\left(\xi|R| / \mu^{2}\right), p=1,2$ ( $\mu$ is the subtraction scale). These generate corrections linear in the scalaron to the "usual" Starobinsky potential and a "running" scalaron mass. Second, for a small fixed Higgs field $\phi^{2} \ll M_{p}^{2} / \xi$ and a vanishing classical coefficient of the $R^{2}$ term, we show that "usual" Starobinsky inflation is generated by quantum corrections alone, for a suitable nonminimal coupling $(\xi)$.
\end{abstract}

DOI: 10.1103/PhysRevD.98.103524

\section{INTRODUCTION}

The idea of inflation in the early universe [1-8] (for a review [9]) led to many models in agreement with the cosmic microwave background CMB [10]; of these, minimal models like Starobinsky model [2] and the Higgs inflation model [11-13] are among the most successful (for more recent developments in Higgs inflation, see, e.g., [14-25] and in the $R^{2}$ models [26-38]).

In Higgs inflation, a nonminimal coupling $\xi \phi^{2} R$ of the Higgs $\phi$ to the Ricci scalar $R$ is considered, with $\phi$ also in the role of the inflaton. This relates cosmology to Standard Model precision tests. In Starobinsky inflation an $\alpha R^{2}$ term ( $\alpha$ constant) is added to the Einstein term, thus inducing geometrically a new scalar field $\rho$ (scalaron) playing the role of inflaton. Both models give a similar spectral index of primordial scalar adiabatic perturbations.

If quantum corrections of matter are included, these two models are special limits of a single model of inflation (hereafter "Starobinsky-Higgs"). Indeed, consider the quantum corrections in curved spacetime due to $\phi$ to the Higgs potential $V_{0}(\phi)$ of flat spacetime. New terms linear

\footnotetext{
*dumitru.ghilencea@cern.ch
}

Published by the American Physical Society under the terms of the Creative Commons Attribution 4.0 International license. Further distribution of this work must maintain attribution to the author(s) and the published article's title, journal citation, and DOI. Funded by SCOAP ${ }^{3}$. in $R$, e.g., $V_{1}(\phi) \sim R \phi^{2} \ln \phi$ and quadratic in $R$, Ricci $\left(R_{\mu \nu}\right)$ or Riemann $\left(R_{\mu \nu \rho \sigma}\right)$ tensors, e.g., $V_{2}(\phi) \sim R^{2} \ln \phi$, emerge in the quantum action with $\phi$-dependent coefficients, even if they are absent at tree level. $R^{2}$-inflation and Higgs inflation are thus unified in a single quantum model of fields $(\phi, \rho)$, so multifield inflation is a quantum consequence.

The main goal is to study the effects of quantum corrections of matter to the Starobinsky-Higgs model. This study is useful for precision tests of this model. With either the Higgs or the $R^{2}$ term in the action, one can achieve successful inflation. Their "overlap" in the general parameter region of Starobinsky-Higgs inflation is then not expected to give dramatic deviations from $R^{2}$-inflation or Higgs inflation alone. One linear combination $\phi$-scalaron is essentially responsible for inflation; its quantum fluctuations are adiabatic perturbations becoming the seeds of inhomogeneities seen in the CMB temperature anisotropy. The other combination gives rise to isocurvature perturbations [39] specific to multifield inflation.

The plan of the paper is as follows. We first review at the classical level the Starobinsky-Higgs inflation (Sec. II). For special values of the fields and $\xi, \alpha$ (coefficient of $R^{2}$ ) and $\lambda$ (Higgs self-coupling), one has Higgs inflation, $R^{2}$ inflation, or a combination of these. We study the two-loop corrections to the effective action with nonminimal coupling $\xi$ and quadratic curvature terms $\left(R^{2}, R_{\mu \nu}^{2}, R_{\mu \nu \rho \sigma}^{2}\right)$, following [25,40-44] (Sec. III). The metric is not quantized. 
One expands the potential in powers of curvature [41] and solves iteratively the two-loop Callan-Symanzik equations for $V_{0}(\phi), V_{1}(\phi), V_{2}(\phi)$ (Appendix). We then study the Einstein frame potential $\hat{W}(\phi, \rho)$ which also depends on the scalaron. The effects of the quantum corrections to the classical potential and the spectral index $n_{s}$ are analyzed. In general the Starobinsky-Higgs model is stable in their presence (Sec. III D).

Two special cases are also studied. At large $\xi$, integrating the Higgs field in the quantum action generates a "refined" Starobinsky model with extra terms $\xi^{2} R^{2} \ln ^{n}\left(\xi|R| / \mu^{2}\right)$, $n=1,2$; these bring terms $\propto \rho$ and a running scalaron mass. Terms like $C^{2} \ln |R| / \mu^{2}$ and $G \ln |R| / \mu^{2}$ are also generated, with $C^{2}$ the square of Weyl tensor, $G$ : GaussBonnet term (Sec. III B). Finally, we show that even if there is no $R^{2}$ term at the tree level $(\alpha=0)$, a term $(\xi+1 / 6)^{2} R^{2} \ln \phi$ emerges at the loop level. This is an interesting result, since the mere presence of a fixed, small Higgs field $\phi \ll M_{p}$ with large nonminimal coupling $\xi \phi^{2} R$, provides a quantum origin to the "usual" Starobinsky model of inflation!

\section{STAROBINSY-HIGGS MODEL: CLASSICAL PICTURE}

Our starting action is that of a scalar theory $\phi^{4}$ in curved spacetime with quadratic terms ${ }^{1}$

$$
\begin{aligned}
S= & \int d^{4} x \sqrt{g}\left\{\frac{1}{2} g^{\mu \nu} \partial_{\mu} \phi \partial_{\nu} \phi-\frac{1}{2} \xi R \phi^{2}-\frac{1}{4 !} \lambda \phi^{4}-\Lambda\right. \\
& \left.-\frac{1}{2} M_{p}^{2} R+\alpha_{1} R_{\mu \nu \rho \sigma} R^{\mu \nu \rho \sigma}+\alpha_{2} R_{\mu \nu} R^{\mu \nu}+\alpha_{3} R^{2}\right\} .
\end{aligned}
$$

$M_{p}=(8 \pi G)^{-1 / 2}, g \equiv\left|\operatorname{det} g_{\mu \nu}\right|$ and $\alpha_{1,2,3}$ and $\xi$ are coupling constants; we also set $\Lambda=0$. We re-write (1) in terms of the (square of) Weyl tensor $\left(C^{2}\right)$ and Gauss-Bonnet term $(G)$ :

$$
\begin{aligned}
R_{\mu \nu \rho \sigma} R^{\mu \nu \rho \sigma} & =2 C^{2}-G+\frac{1}{3} R^{2}, \\
R_{\mu \nu} R^{\mu \nu} & =\frac{1}{2} C^{2}-\frac{1}{2} G+\frac{1}{3} R^{2} .
\end{aligned}
$$

Then

$$
\begin{aligned}
S= & \int d^{4} x \sqrt{g}\left\{\frac{1}{2} g^{\mu \nu} \partial_{\mu} \phi \partial_{\nu} \phi-\frac{1}{2}\left(M_{p}^{2}+\xi \phi^{2}\right) R-\frac{\lambda}{4 !} \phi^{4}\right. \\
& \left.+\alpha R^{2}+\gamma C^{2}+\delta G\right\},
\end{aligned}
$$

\footnotetext{
${ }^{1}$ Our conventions [45]: metric: $(+,-,-,-) ; R_{\mu \nu \sigma}^{\lambda}=-\partial_{\sigma} \Gamma_{\mu \nu}^{\lambda}+$ $\partial_{\nu} \Gamma_{\mu \sigma}^{\lambda}+\cdots, R_{\mu \nu}=R_{\mu \lambda \nu}^{\lambda}, R=g^{\mu \nu} R_{\mu \nu} ; R<0$ at inflationary stage; $\xi=-\frac{1}{6}$ is conformal. To use conventions [41] do $R \rightarrow-R, \xi \rightarrow-\xi, \beta_{\xi} \rightarrow-\beta_{\xi}$.
}

where

$\alpha=\alpha_{3}+\frac{1}{3}\left(\alpha_{1}+\alpha_{2}\right), \quad \gamma=2 \alpha_{1}+\frac{\alpha_{2}}{2}, \quad \delta=-\alpha_{1}-\frac{\alpha_{2}}{2}$.

The Gauss-Bonnet term is topological (total derivative); the Weyl tensor is vanishing in the Friedmann-RobertsonWalker universe. Also, the coefficients $\gamma, \delta$ are independent of $\xi$ and do not play a role in inflation. Depending on the values of the field $\phi$ and of $\xi, \alpha, \lambda$, Eq. (3) covers both Higgs- and Starobinsky-inflation models which are different limits of the same model as we briefly review below (for a review, see [46]).

One eliminates $R^{2}$ in (3) by replacing it with $R^{2} \rightarrow$ $-2 \sigma^{2} R-\sigma^{4}$ with $\sigma$ a new (auxiliary) scalar field; its equation of motion $\sigma^{2}=-R$ recovers (3) from a new, equivalent form

$S=\int d^{4} x \sqrt{g}\left\{\frac{1}{2} g^{\mu \nu} \partial_{\mu} \phi \partial_{\nu} \phi-\frac{1}{2} M_{p}^{2} f(\phi, \sigma) R-W(\phi, \sigma)\right\}$,

where

$$
\begin{aligned}
f(\phi, \sigma) & =1+\frac{1}{M_{p}^{2}}\left[4 \alpha \sigma^{2}+\xi \phi^{2}\right], \\
W(\phi, \sigma) & =\frac{1}{4 !} \lambda \phi^{4}+\alpha \sigma^{4} .
\end{aligned}
$$

Assume $\alpha>0, \xi>0$, therefore $f>0$ and we rescale the metric to $^{2} \hat{g}_{\mu \nu}=f(\phi, \sigma) g_{\mu \nu}$. Then the action becomes (variables in the Einstein frame are marked with a hat):

$$
\begin{aligned}
\hat{S}= & \int d^{4} x \sqrt{\hat{g}}\left\{\frac{1}{2 f} \hat{g}^{\mu \nu}\left(\partial_{\mu} \phi\right)\left(\partial_{\nu} \phi\right)-\frac{1}{f^{2}} W(\phi, \sigma)-\frac{M_{p}^{2}}{2} \hat{R}\right. \\
& \left.+\frac{3}{4} M_{p}^{2} \hat{g}^{\mu \nu} \partial_{\mu}(\ln f) \partial_{\nu}(\ln f)\right\} .
\end{aligned}
$$

After a field redefinition $(\sigma \rightarrow \rho)$ with $\rho$ a new real scalar field:

$$
\ln f(\phi, \sigma)=q_{0} \rho, \quad q_{0} \equiv \frac{\sqrt{2 / 3}}{M_{p}},
$$

then

$$
\begin{aligned}
\hat{S}= & \int d^{4} x \sqrt{\hat{g}}\left\{\frac{1}{2} e^{-q_{0} \rho} \hat{g}^{\mu \nu}\left(\partial_{\mu} \phi\right)\left(\partial_{\nu} \phi\right)-\hat{W}(\phi, \rho)\right. \\
& \left.-\frac{1}{2} M_{p}^{2} \hat{R}+\frac{1}{2} \hat{g}^{\mu \nu}\left(\partial_{\mu} \rho\right)\left(\partial_{\nu} \rho\right)\right\}
\end{aligned}
$$

\footnotetext{
${ }^{2}$ We use $\int d^{4} x \sqrt{g}(1 / 2) f R=\int d^{4} x \sqrt{\hat{g}}(1 / 2)\left[\hat{R}-(3 / 2) \hat{g}^{\mu \nu}\right.$ $\left.\partial_{\mu}(\ln f) \partial_{\nu}(\ln f)\right]$.
} 
with

$$
\begin{aligned}
\hat{W}(\phi, \rho) & =\frac{3}{4} M_{p}^{2} M_{\alpha}^{2}\left[1-\left(1+\frac{\xi \phi^{2}}{M_{p}^{2}}\right) e^{-q_{0} \rho}\right]^{2}+\frac{\lambda}{4 !} \phi^{4} e^{-2 q_{0} \rho}, \\
M_{\alpha}^{2} & =\frac{M_{p}^{2}}{12 \alpha}
\end{aligned}
$$

where scalaron $\rho$ enters as an exponent only. Equations (9), (10) give the Einstein frame result.

One has Starobinsky inflation if $\lambda=\xi=0$ (Higgs field absent) or $\xi \phi^{2} \ll M_{p}^{2}$ and $\alpha \sim 5 \times 10^{8}$ [47-49]; then $\exp \left(q_{0} \rho\right)=1+4 \alpha \sigma^{2} / M_{p}^{2}$. For large $\sigma$, the first kinetic term vanishes and

$$
\hat{W}=\frac{3}{4} M_{p}^{2} M_{\alpha}^{2}\left(1-e^{-q_{0} \rho}\right)^{2}, \quad M_{\alpha}^{2}=\frac{M_{p}^{2}}{12 \alpha} .
$$

One has Higgs inflation if $\alpha=0\left(M_{\alpha} \rightarrow \infty\right)$ or $\alpha \sigma^{2} \ll$ $M_{p}^{2}$ with $\xi \sim 1.8 \times 10^{4}[12]$. Then $\exp \left(q_{0} \rho\right)=1+\xi \phi^{2} / M_{p}^{2}$. For large $\phi$, the first kinetic term vanishes and the potential is

$$
\hat{W}=\frac{3}{4} M_{p}^{2} M_{\xi}^{2}\left(1-e^{-q_{0} \rho}\right)^{2}, \quad M_{\xi}^{2}=\frac{\lambda}{18 \xi^{2}} M_{p}^{2} .
$$

Finally, consider the limit of large $\xi$ with $\alpha \neq 0$. In the Jordan frame, the Higgs field kinetic term is subleading to $\xi \phi^{2} R$ and can be ignored (at least during inflation). Then $\phi$ can be integrated out via its equation of motion which is $\phi_{c}^{2}=-6 \xi R / \lambda$. Then Eq. (3) gives [28]

$$
\begin{aligned}
S & =\int d^{4} x \sqrt{g}\left\{-\frac{1}{2} M_{p}^{2} R+\frac{M_{p}^{2}}{12 M^{2}} R^{2}+\cdots\right\}, \\
M^{2} & =\frac{M_{p}^{2}}{12\left(\alpha+3 \xi^{2} /(2 \lambda)\right)} .
\end{aligned}
$$

This is the "usual" Starobinsky model, which in the Einstein frame gives Eq. (11) with $\alpha \rightarrow \alpha+\frac{3 \xi^{2}}{(2 \lambda)}$ and a modified scalaron mass $M \neq M_{\alpha}$. The relation between $M$ and $M_{\alpha}$ is

$$
M^{2}=\frac{M_{\alpha}^{2}}{1+18\left(\xi^{2} / \lambda\right) M_{\alpha}^{2} / M_{p}^{2}}
$$

To reproduce the observed amplitude of curvature perturbations $M \approx 1.3 \times 10^{-5} M_{p}$ [49]; this brings a correlation among $\xi, \lambda$ and $\alpha$; if $\xi=\sqrt{\lambda / 18}\left(M_{p} / M\right), M$ fixed, then $M_{\alpha} \rightarrow \infty$.

\section{STAROBINSKY-HIGGS MODEL: QUANTUM CORRECTIONS}

\section{A. Two-loop effective action with nonminimal coupling and $\boldsymbol{R}^{2}$ terms}

Consider now the quantum corrections to action (1); this is analytically continued to $d=4-2 \epsilon$ dimensions, for regularization purposes. The "bare" action has a similar form. Note that the metric is not quantized. The bare $\left(L_{B}\right)$ and renormalized $(L)$ Lagrangian are related by $L_{B}=\mu^{2 \epsilon} L$. At two-loop the counterterms $\delta L$ have the same form as (1) $\left(\delta \Lambda=\delta M_{p}=0\right)$.

The quantum corrected potential $V$ is found by using its expansion in powers of the curvature and its CallanSymanzik (CS) equation:

$\left(\mu \frac{\partial}{\partial \mu}+\beta_{\lambda} \frac{\partial}{\partial \lambda}+\beta_{\xi} \frac{\partial}{\partial \xi}-\gamma \phi \frac{\partial}{\partial \phi}+\sum_{j=1}^{3} \beta_{\alpha_{j}} \frac{\partial}{\partial \alpha_{j}}\right) V(\phi)=0$,

where $\beta_{X}$ is the beta function of the coupling $X$ and $\gamma$ is the anomalous dimension of $\phi$.

The two-loop solution to (15) with terms quadratic in curvature is found by using the method of $[25,41]$. Then

$$
V(\phi)=V_{0}(\phi)+V_{1}(\phi)+V_{2}(\phi) .
$$

$V_{0}$ is the potential in flat spacetime, $V_{1}$ is linear in $R, V_{2}$ contain terms quadratic $R, R_{\mu \nu}$ and $R_{\mu \nu \rho \sigma}$. We ignore higher powers of $R$, etc. From Eq. (15), by grouping terms of similar structure (linear in $R$, etc.), one finds three independent CS equations for $V_{0}, V_{1}, V_{2}$. These are solved iteratively at two-loop (order by order); see Appendix A for details. One has

$V_{0}=\frac{\tilde{\lambda}(\phi)}{4 !} \phi^{4}, \quad V_{1}=\frac{\tilde{\xi}(\phi)}{2} R \phi^{2}$,

$V_{2}=-\tilde{\alpha}_{1}(\phi) R_{\mu \nu \rho \sigma} R^{\mu \nu \rho \sigma}-\tilde{\alpha}_{2}(\phi) R_{\mu \nu} R^{\mu \nu}-\tilde{\alpha}_{3}(\phi) R^{2}$.

$\tilde{\lambda}, \tilde{\xi}$ and $\tilde{\alpha}_{1,2,3}$ are functions ${ }^{3}$ of $\phi$ :

$$
\tilde{\lambda}=\tilde{\lambda}(\phi), \quad \tilde{\xi}=\tilde{\xi}(\phi), \quad \tilde{\alpha}_{i}=\tilde{\alpha}_{i}(\phi) .
$$

At two-loop level, from (A13),

$$
\begin{aligned}
\tilde{\lambda}= & \lambda\left\{1+\frac{3 \lambda}{2 \kappa}\left[\ln \frac{\phi^{2}}{\mu^{2}}-\frac{25}{6}\right]\right. \\
& \left.+\frac{3 \lambda^{2}}{4 \kappa^{2}}\left[\frac{515}{6}-29 \ln \frac{\phi^{2}}{\mu^{2}}+3 \ln ^{2} \frac{\phi^{2}}{\mu^{2}}\right]\right\},
\end{aligned}
$$

\footnotetext{
${ }^{3}$ To simplify notation, below we do not write explicitly their argument $\phi$.
} 
with $\kappa=(4 \pi)^{2}$. From (A22)

$$
\begin{aligned}
\frac{\tilde{\xi}}{2}= & \left\{\frac{\xi}{2}+\frac{\lambda}{4 \kappa}\left(\xi+\frac{1}{6}\right)\left(\ln \frac{\phi^{2}}{\mu^{2}}-3\right)\right. \\
& -\frac{\lambda^{2}}{4 \kappa^{2}}\left(\frac{7}{6} \xi+\frac{13}{36}\right)\left(\ln \frac{\phi^{2}}{\mu^{2}}-3\right) \\
& \left.+\frac{2 \lambda^{2}}{\kappa^{2}} \frac{1}{4}\left(\xi+\frac{1}{6}\right)\left(7-3 \ln \frac{\phi^{2}}{\mu^{2}}+\frac{1}{2} \ln ^{2} \frac{\phi^{2}}{\mu^{2}}\right),\right\}
\end{aligned}
$$

and finally, from Eqs. (A24), (A31),

$$
\begin{aligned}
& -\tilde{\alpha}_{j}=-\alpha_{j}+(-1)^{j+1} \frac{1}{180 \kappa} \ln \frac{\phi}{\mu}, \quad j=1,2 ; \\
& -\tilde{\alpha}_{3}=-\alpha_{3}+\frac{1}{2 \kappa}\left(\xi+\frac{1}{6}\right)^{2} \ln \frac{\phi}{\mu}+\frac{\lambda}{2 \kappa^{2}}\left(\xi+\frac{1}{6}\right)^{2} \ln ^{2} \frac{\phi}{\mu} .
\end{aligned}
$$

To solve for $V_{0}, V_{1}, V_{2}$, the following boundary conditions were used

$$
\begin{aligned}
\left.V_{0}^{(4)}\right|_{t=0} & =\lambda,\left.\quad V_{1}^{(2)}\right|_{t=0}=R \xi \\
\left.\tilde{\alpha}_{j}\right|_{t=0} & =\alpha_{j},(j: 1,2,3), \quad t \equiv \ln \frac{\mu}{\phi}
\end{aligned}
$$

Here $V_{j}^{(k)}$ is the $k$-th derivative of $V_{j}$ with respect to $\phi$. Note that $\tilde{\xi}, \tilde{\lambda}, \tilde{\alpha}_{j}$ are all functions of $t$ and that $V$ simplifies in the one-loop conformal limit of $\xi=-1 / 6$.

With the above expressions the action becomes

$$
\begin{aligned}
S= & \int d^{d} x \sqrt{g}\left\{\frac{1}{2} Z_{\phi} g^{\mu \nu} \partial_{\mu} \phi \partial_{\nu} \phi-\frac{1}{2}\left(M_{p}^{2}+\tilde{\xi} \phi^{2}\right) R-\frac{\tilde{\lambda}}{4 !} \phi^{4}\right. \\
& \left.+\tilde{\alpha}_{1} R_{\mu \nu \rho \sigma} R^{\mu \nu \rho \sigma}+\tilde{\alpha}_{2} R_{\mu \nu} R^{\mu \nu}+\tilde{\alpha}_{3} R^{2}\right\} .
\end{aligned}
$$

Using Eq. (2), the $R^{2}$ term acquires an extra correction

$$
\begin{aligned}
S= & \int d^{d} x \sqrt{g}\left\{\frac{1}{2} Z_{\phi}\left(\partial_{\mu} \phi\right)^{2}-\frac{1}{2}\left(M_{p}^{2}+\tilde{\xi} \phi^{2}\right) R\right. \\
& \left.-\frac{\tilde{\lambda}}{4 !} \phi^{4}+\tilde{\alpha} R^{2}+\tilde{\gamma} C^{2}+\tilde{\delta} G\right\},
\end{aligned}
$$

where

$$
\tilde{\alpha}=\tilde{\alpha}_{3}+\frac{1}{3}\left(\alpha_{1}+\alpha_{2}\right), \quad \tilde{\gamma}=2 \tilde{\alpha}_{1}+\frac{\tilde{\alpha}_{2}}{2}, \quad \tilde{\delta}=-\tilde{\alpha}_{1}-\frac{\tilde{\alpha}_{2}}{2}
$$

The action in (24), (25) is a combination of the Higgs and Starobinsky inflationary models. Here $\tilde{\xi}, \tilde{\alpha}$ and $\tilde{\lambda}$ are field-dependent, two-loop corrected couplings, also $\tilde{\gamma}, \tilde{\delta}$ acquired $\phi$-dependence but remain $\xi$ independent; their $\phi$ dependence may still play a role, e.g., via equations of motion, if one integrates $\phi$.

Consider now the limit of (24) when the $R^{2}$ term has a vanishing classical coupling $\alpha=0$; see (4). With a subtraction scale $\mu \sim M_{p}$ and $\phi \ll M_{p}$, then $\tilde{\alpha}(\phi)>0$ and

$$
\tilde{\alpha}(\phi)=\frac{1}{2 \kappa}\left(\xi+\frac{1}{6}\right)^{2} \ln \frac{M_{p}}{\phi}+\text { two - loop. }
$$

For $\phi$ fixed, small enough $\phi_{0}^{2} \ll M_{p}^{2} / \tilde{\xi}$, action (24) is dominated by: $(1 / 2) M_{p}^{2} R+\tilde{\alpha}\left(\phi_{0}\right) R^{2}$.

Therefore, we find an interesting result: even if it is absent at the classical level, the "usual" Starobinsky inflation is generated at the quantum level, if there exists a suitable (large) nonminimal coupling $\xi$ of the Higgs field (which otherwise plays no role in inflation), see also [30]. This requires tuning $\xi$ (rather than $\alpha$ [48]), with $\tilde{\xi} \sim \xi$ for small $\lambda$.

\section{B. Large $\xi$ limit: Scalaron quantum action after integrating $\phi$}

Let us consider the limit of large $\xi$ in the quantum action Eq. (24), integrate $\phi$ analytically and then find the effective action. This is similar to the classical discussion near Eq. (13); at large $\xi$ the kinetic term of $\phi$ is subleading to similar two-derivative terms coming from $\xi \phi^{2} R$ after integration by parts and can be ignored. Then $\phi$ is integrated via its equation of motion $\phi_{c}^{2}=6 \xi|R| / \lambda$. One can still use $\phi_{c}$ at the one-loop level, as we do below.

After integrating $\phi$, the action in (24) becomes (ignoring hereafter the $C^{2}$ and $G$ terms)

$$
S=\int d^{4} x \sqrt{g}\left\{\frac{1}{2} M_{p}^{2} F(-R)\right\}
$$

where

$$
F(R)=R+\frac{2 R^{2}}{M_{p}^{2}} \gamma_{0}\left[1+\gamma_{1}+\gamma_{2} \ln \frac{6 \xi|R|}{\lambda \mu^{2}}+\gamma_{3} \ln ^{2} \frac{6 \xi|R|}{\lambda \mu^{2}}\right]
$$

and where $\gamma_{0,1,2,3}$ are read from (19), (20), (21). At one loop,

$\gamma_{0}=\alpha+3 \xi^{2} /(2 \lambda), \quad \gamma_{1}=\frac{3 \xi(13 \xi-2)}{8 \kappa \gamma_{0}}$,
$\gamma_{2}=-\frac{1}{\kappa \gamma_{0}}\left(\xi^{2}-\xi / 6+1 / 144\right), \quad \gamma_{3}=0$.

Note the $R^{2} \ln |R|$ term generated by integrating matter field $\phi ; R^{2} \ln ^{2}|R|$ terms are also generated at two loops. Integrating $\phi$ in (24) also induces a "mixing," ignored 
below, $\left(-C^{2}+G / 3\right) \ln \left(\xi|R| / \mu^{2}\right)$ since $\tilde{\gamma}, \tilde{\delta}$ depend on $\phi$ (see also [50]). From (28) for $\xi^{2} \gg \lambda \alpha$,

$$
\begin{aligned}
S= & \int d^{4} x \sqrt{g}\left\{-\frac{1}{2} M_{p}^{2} R+\frac{M_{p}^{2}}{12 M^{2}} R^{2}\left[1+\frac{13 \lambda}{4 \kappa}\right.\right. \\
& \left.\left.-\frac{2 \lambda}{3 \kappa} \ln \frac{(6 \xi|R|)}{\lambda \mu^{2}}\right]+\mathcal{O}\left(\alpha \lambda / \xi^{2}\right)\right\},
\end{aligned}
$$

with $M$ as in (13). Equations (27) through (30) give a oneloop corrected version of the classical action in (13). A similar action was analyzed recently for cosmological consequences $^{4}$ in [52-56],

$$
S=\int d^{4} \sqrt{g}\left\{-\frac{1}{2} M_{p}^{2} R+\frac{a}{2} R^{2} \frac{1}{\left[1+b \ln \left(|R| / \mu^{2}\right)\right]}\right\},
$$

and it was found that successful inflation demands $a \sim$ $10^{8}-10^{9}$ and $b \leq 10^{-2}$ (for the spectral index to agree with observations) [52]. In our case, $a=2 \gamma_{0}\left[1+\gamma_{1}+\ln (6 \xi / \lambda)\right]$ and $b=-\gamma_{2} /(1+\ln (6 \xi / \lambda))$. If $\xi^{2} \gg \lambda \alpha$, then $a=3 \xi^{2} / \lambda$ and $b=2 \lambda /(3 \kappa) \approx 4.2 \times 10^{-3} \lambda$. The constraint for $a$ is respected by suitable $\xi \sim 10^{4} \sqrt{\lambda}$, while that of $b$ is easily satisfied for perturbative $\lambda$. Increasing $b$, which is of quantum origin here, leads to an increase of the scalarto-tensor ratio [52]. From $b$, one also can infer constraints on the matter content.

To find the Einstein frame potential, Eqs. (27), (30) can be written as [57]

$$
S=\int d^{4} x \sqrt{g} \frac{1}{2} M_{P}^{2}\left[-F^{\prime}(\sigma) R+F(\sigma)-\sigma F^{\prime}(\sigma)\right] .
$$

The equation of motion for auxiliary field $\sigma$ is $\sigma=-R>0$ and if used in (32) recovers (27). Further, in (32), we rescale the metric followed by a field redefinition $\sigma \rightarrow \rho$, using $\hat{g}_{\mu \nu}=F^{\prime}(\sigma) g_{\mu \nu}$ and $\ln F^{\prime}(\sigma)=q_{0} \rho$ where, as usual, $q_{0} \equiv \sqrt{(2 / 3)}\left(1 / M_{p}\right)$; also, $F^{\prime}(\sigma)>0$. We then find the action for $\rho$ in the Einstein frame,

$$
\hat{S}=\int d^{4} x \sqrt{\hat{g}}\left\{-\frac{1}{2} M_{p}^{2} \hat{R}+\frac{1}{2} \hat{g}^{\mu \nu} \partial_{\mu} \rho \partial_{\nu} \rho-\hat{W}_{\mathrm{eff}}(\rho)\right\}
$$

with

$$
\hat{W}_{\mathrm{eff}}(\rho)=\frac{1}{2} M_{p}^{2} \frac{\sigma}{F^{\prime}(\sigma)}\left\{1-\frac{1}{\sigma} \frac{F(\sigma)}{F^{\prime}(\sigma)}\right\}_{\sigma=-R(\rho)}
$$

\footnotetext{
${ }^{4}$ For a similar action, of different, quantum gravitational origin, see [51].
}

As shown in (34), $\sigma$ is replaced by the solution of the equation: $\ln F^{\prime}(-R)=q_{0} \rho$. To find the solution $R(\rho)$, we search for one of the form

$$
\begin{aligned}
R & =R_{0}\left[1+\delta_{1}+\delta_{2} \ln \frac{6 \xi\left|R_{0}\right|}{\lambda \mu^{2}}\right], \\
R_{0} & \equiv-\frac{M_{p}^{2}}{4 \gamma_{0}}\left(e^{q_{0} \rho}-1\right) .
\end{aligned}
$$

$R_{0}$ is the solution at the tree level, in which $\mathcal{O}\left(1 / \kappa^{n}\right)$ terms, $n \geq 1$ are ignored. Using (28) we find $\delta_{1}=-\left(\gamma_{1}+\gamma_{2} / 2\right)$, $\delta_{2}=-\gamma_{2}$. With (35), the scalaron effective potential is

$$
\begin{aligned}
\hat{W}_{\text {eff }}(\rho)= & \frac{3}{4} M_{p}^{2} M^{2}\left[1-e^{-q_{0} \rho}\right]^{2}\left\{1-\gamma_{1}-\gamma_{2}\left[\ln \left(\frac{3 \xi M_{p}^{2}}{2 \lambda \gamma_{0} \mu^{2}}\right)\right.\right. \\
& \left.\left.+q_{0} \rho+\ln \left[1-e^{-q_{0} \rho}\right]\right]\right\},
\end{aligned}
$$

with $M$ defined in Eq. (13). Notice the correction linear in $\rho$. The potential contains a dependence on the subtraction scale $\mu$, as expected. Further, if $\xi^{2} \gg \lambda \alpha$, then

$$
\begin{aligned}
\hat{W}_{\mathrm{eff}}(\rho)= & \frac{3}{4} M_{p}^{2} M_{\mu}^{2}\left[1-e^{-q_{0} \rho}\right]^{2}\left\{1-\frac{13 \lambda}{4 \kappa}+\frac{2 \lambda}{3 \kappa} q_{0} \rho\right. \\
& \left.+\frac{2 \lambda}{3 \kappa} \ln \left[1-e^{-q_{0} \rho}\right]\right\},
\end{aligned}
$$

with

$$
M_{\mu}^{2}=M^{2}\left[1+\frac{2 \lambda}{3 \kappa} \ln \frac{M_{p}^{2} / \xi}{\mu^{2}}\right] .
$$

Equation (37) is the Einstein frame counterpart to Eq. (30). The dependence of $\hat{W}_{\text {eff }}$ on the scale $\mu$ was included in $M_{\mu}$; this may be regarded as the "running" scalaron mass, with an "emergent" UV cutoff $M_{p} / \sqrt{\xi}$ in the Einstein frame. The new term linear in scalaron $(\propto \rho)$ is important near/ above the Planck scale $M_{p}$ but its effect is reduced by a small $\lambda$ (of the Standard Model). These corrections correspond to the $R^{2} \ln \left(\xi|R| / \mu^{2}\right)$ term in the Jordan frame. In the absence of these terms (e.g., if $\lambda \rightarrow 0$ ) we recover the "standard" Starobinsky inflation. This analysis can be repeated in the two-loop action, using a one-loop solution of the equation of motion of $\phi$.

\section{Einstein frame action}

The general two-loop result in Eq. (24) can be mapped to the Einstein frame. We eliminate the $R^{2}$ dependence via a replacement $R^{2} \rightarrow-2 \sigma^{2} R-\sigma^{4}$ in (25) where $\sigma$ is a new real scalar field. Its equation of motion $\sigma^{2}=-R$ recovers (24) from a new, equivalent action: ${ }^{5}$

\footnotetext{
${ }^{5}$ We ignore here the additional terms $\tilde{\gamma} C^{2}+\tilde{\delta} G$ in (24).
} 
$S=\int d^{d} x \sqrt{g}\left\{\frac{1}{2} Z_{\phi}\left(\partial_{\mu} \phi\right)^{2}-\frac{1}{2} M_{p}^{2} \tilde{f}(\phi, \sigma) R-\tilde{W}(\phi, \sigma)\right\}$,

where

$$
\begin{aligned}
& \tilde{f}(\phi, \sigma)=1+\frac{1}{M_{p}^{2}}\left[4 \tilde{\alpha} \sigma^{2}+\tilde{\xi} \phi^{2}\right], \\
& \tilde{W}(\phi, \sigma)=\frac{1}{4 !} \tilde{\lambda} \phi^{4}+\tilde{\alpha} \sigma^{4}
\end{aligned}
$$

Avoiding ghosts requires $\tilde{f}>0$, which is true if $\xi, \alpha>0$ as assumed (the determinant of the matrix in field space $\phi, \sigma$ is then positive). We follow previous steps and rescale the metric to $\hat{g}_{\mu \nu}=\tilde{f}(\phi, \sigma) g_{\mu \nu}$, then redefine $\ln \tilde{f}(\phi, \sigma)=q_{0} \rho$ with $q_{0} \equiv \sqrt{(2 / 3)} / M_{p}$. Then

$$
\begin{aligned}
\hat{S}= & \int d^{d} x \sqrt{\hat{g}}\left\{\frac{1}{2} Z_{\phi} e^{-q_{0} \rho} \hat{g}^{\mu \nu}\left(\partial_{\mu} \phi\right)\left(\partial_{\nu} \phi\right)-\hat{W}(\phi, \rho)\right. \\
& \left.-\frac{1}{2} M_{p}^{2} \hat{R}+\frac{1}{2} \hat{g}^{\mu \nu}\left(\partial_{\mu} \rho\right)\left(\partial_{\nu} \rho\right)\right\},
\end{aligned}
$$

with

$$
\begin{aligned}
\hat{W}= & \frac{3}{4} M_{p}^{2} \tilde{M}(\phi)^{2}\left[1-\left(1+\frac{\tilde{\xi}(\phi) \phi^{2}}{M_{p}^{2}}\right) e^{-q_{0} \rho}\right]^{2} \\
& +\frac{\tilde{\lambda}(\phi)}{4 !} \phi^{4} e^{-2 q_{0} \rho} \\
\tilde{M}^{2}(\phi)= & \frac{M_{p}^{2}}{12 \tilde{\alpha}(\phi)}
\end{aligned}
$$

in the Einstein frame. We made explicit the $\phi$ dependence of $\tilde{M}, \tilde{\xi}, \tilde{\lambda}, \tilde{\alpha}$. As at tree level, $\rho$ shows up only as an exponent that suppresses the kinetic term of $\phi$.

Equations (41), (42) are the counterpart to the Jordan frame quantum result of Eq. (24), in terms of $\tilde{M}, \tilde{\xi}, \tilde{\lambda}$; see also the classical version, Eqs. (9), (10). Either the Higgs field or scalaron or their mixing plays the inflaton role, depending on the relative size of these couplings at quantum level. We thus have a standard two-field inflationary model, see e.g., [58], with diagonal metric $\gamma_{a b}$ in the field space $(\phi, \rho)$ but with $\gamma_{\phi \phi}=e^{-q_{0} \rho}$ and $\gamma_{\rho \rho}=1$.

Finally, consider a special case of $\alpha=0$, i.e., no classical $R^{2}$ term. With $\phi$ fixed $\phi=\phi_{0}$ and small enough $\tilde{\xi} \phi_{0}^{2} \ll M_{p}^{2}$, then from (42),

$$
\begin{aligned}
\hat{W} & =\frac{3}{4} M_{p}^{2} \tilde{M}\left(\phi_{0}\right)^{2}\left[1-e^{-q_{0} \rho}\right]^{2}, \\
\tilde{M}\left(\phi_{0}\right)^{2} & \approx \frac{M_{p}^{2}}{12\left(\xi^{2} / \kappa\right) \ln \left(\mu^{2} / \phi_{0}^{2}\right)} .
\end{aligned}
$$

With subtraction scale $\mu$ chosen of order $M_{P}$ and $\phi_{0} \ll \mu$, Eq. (43) gives a quantum origin to $\tilde{M}\left(\phi_{0}\right)$ and to Starobinsky inflation if $\left(\xi^{2} / \kappa\right) \ln \mu^{2} / \phi_{0}^{2} \sim 5 \times 10^{8}$ or $\xi \approx$ $3.5 \times 10^{4}\left(\phi_{0} \sim m_{Z}\right)$, (which is also that needed for Higgs inflation). For this to happen, the nonminimal coupling $\xi$ is essential. This is the Einstein-frame counterpart to the Jordan frame result, Eq. (26).

\section{The behavior of the quantum potential}

Let us examine the potential in Eq. (42) and compare it against its classical behavior [28]. The Hubble parameter is ${ }^{6}$

$$
H^{2}=\frac{1}{3 M_{p}^{2}}\left(\frac{1}{2} \dot{\phi}^{2} e^{-q_{0} \rho}+\frac{1}{2} \dot{\rho}^{2}+\hat{W}\right),
$$

and

$$
\dot{H}=-\frac{1}{2 M_{p}^{2}}\left(\dot{\phi}^{2} e^{-q_{0} \rho}+\dot{\rho}^{2}\right) .
$$

One has slow-roll conditions $\epsilon \ll 1, \eta_{\|} \ll 1$ and spectral index $n_{s}=1+2 \eta_{\|}-4 \epsilon$ [28], where

$$
\begin{aligned}
\epsilon & \equiv-\frac{\dot{H}}{H^{2}}=\frac{\left(\dot{\phi}^{2} e^{-q_{0} \rho}+\dot{\rho}^{2}\right)}{2 M_{p}^{2} H^{2}}, \\
\eta_{\|} & \equiv \frac{-\ddot{\phi}_{0}}{H \dot{\phi}_{0}}, \quad \text { with } \quad \dot{\phi}_{0}^{2} \equiv \gamma_{a b} \dot{\phi}^{a} \dot{\phi}^{b}, \quad(a, b: \phi, \rho) .
\end{aligned}
$$

For slow roll regime and with $\hat{W}$ of (42), one has $H^{2} \approx 1 /\left(3 M_{p}^{2}\right) \hat{W}$. Then for larger values of the field $\rho$ and large $\tilde{\xi}(\phi) \phi^{2}>M_{p}^{2}$ the bracket ${ }^{7}$ in $\hat{W}$ that multiplies $e^{-q_{0} \rho}$ is approximated by the term $\propto \tilde{\xi}$. To compensate for this effect and to keep $H$ constant (so that the amplitude of curvature perturbations is unchanged), one has to increase $\tilde{M}(\phi)$. This is possible by considering a smaller $\tilde{\alpha}(\phi)$ in Eq. (42). Therefore $\tilde{\xi}$ and $\tilde{\alpha} \sim \alpha$ play somewhat opposite roles while keeping $H$ unchanged, and they encode the effects of $\phi$ and $\rho$, respectively; $\tilde{\xi}$ and $\tilde{\alpha}$ are largely controlled by their tree-level values, $\xi$ and $\alpha$, and to a smaller extent by $\phi, \lambda$ and the renormalization scale $\mu$ entering in the quantum corrections of Eqs. (19)-(21).

As apparent in the plots of Fig. 1 where quantum corrections are included, increasing $\xi$ (and $\tilde{\xi}$ ) brings deeper valleys and also changes their initial direction [relative to plot (a1)], while a smaller $\alpha(\tilde{\alpha})$ or larger $\tilde{M}$ increases the height of the potential in its central region (of small $\phi$ ). The actual inflaton roll is along a trajectory in the plane $(\phi, \rho)$

\footnotetext{
${ }^{6}$ In general $H^{2}=1 /\left(3 M_{p}^{2}\right)\left(\frac{1}{2} \gamma_{a b} \dot{\phi}^{a} \dot{\phi}^{b}+W\right), \epsilon=\dot{\phi}_{0}^{2} /\left(2 M_{p}^{2} H^{2}\right)$, $\phi_{0}^{2} \equiv \gamma_{a b} \dot{\phi}^{a} \dot{\phi}^{b}$.

${ }^{7}$ For the other limit, of large $\rho$ and small $\tilde{\xi}, \quad \tilde{W} \approx$ $(3 / 4) M_{p}^{2} \tilde{M}(\phi)^{2} \approx(3 / 4) M_{p}^{2} /(12 \alpha) \approx$ constant.
} 
that is controlled by: the height of the potential (influenced by $\alpha$ ), the position/depth of the valleys (influenced by $\xi$ ), by $H$ and by the field-space metric since $\gamma_{\phi \phi}=$ $\exp \left(-\sqrt{2 / 3} \rho / M_{p}\right)$. The fields then oscillate about the global minimum at $(\phi, \rho)=(0,0)$, where reheating takes place after inflation ends.

Figure 1 shows a behavior of the potential at the quantum level that is similar to the classical one analyzed recently in
[28]. The difference between the classical picture [28] and our quantum picture is usually small; see Fig. 2 for details. In this figure, it was shown that the quantum-corrected $\hat{W}$ is reduced relative to its classical value, by up to $6 \%$ for $\lambda=0.6$, and up to $10 \%$ for $\lambda=1$ (with our normalization of $\lambda$ ). In all these figures, the subtraction scale was set $\mu=M_{p}$. These results change mildly, e.g., by $5 \%$ when reducing $\mu$ by a factor of 100 at $\lambda=0.6$ (due to a $\log \mu$

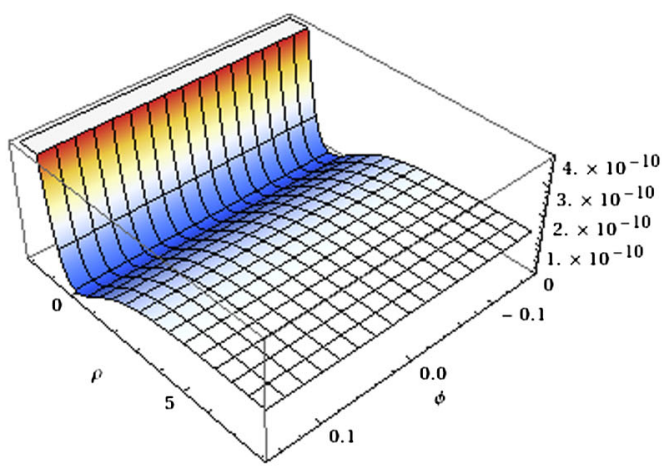

(a1)

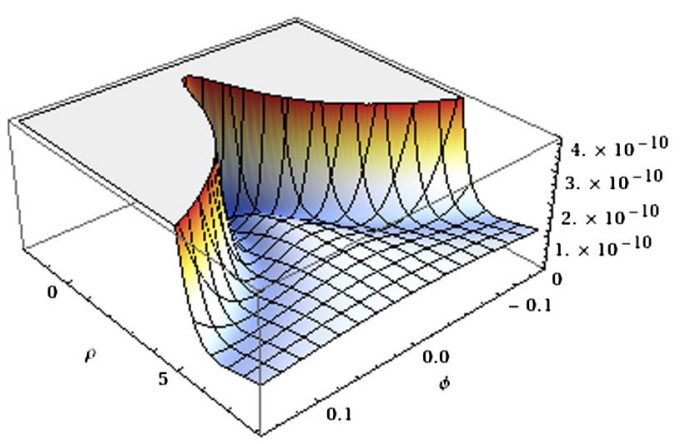

(b1)

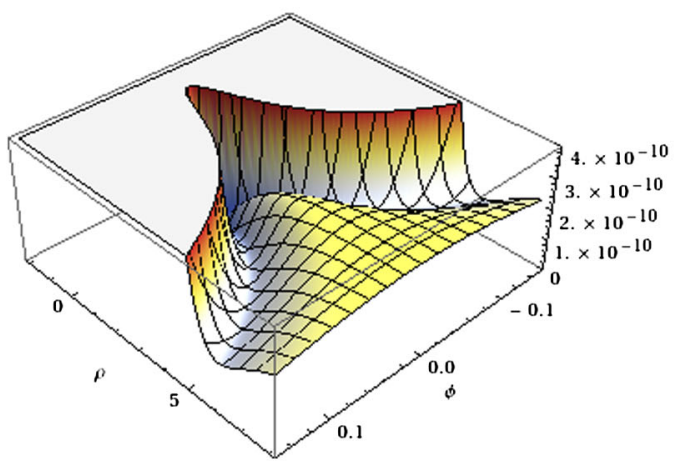

(c1)

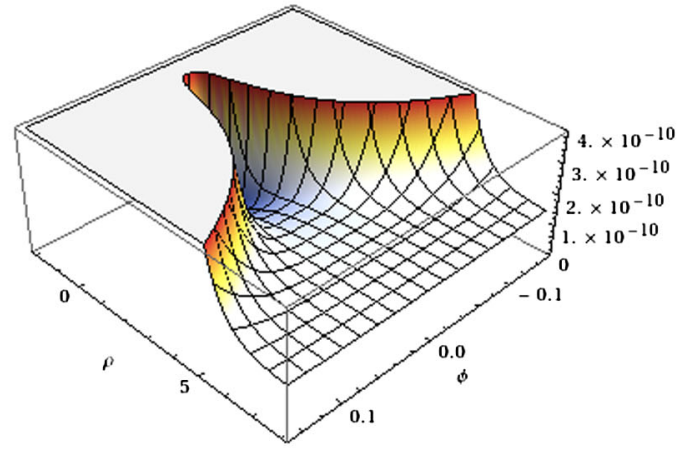

(a2)

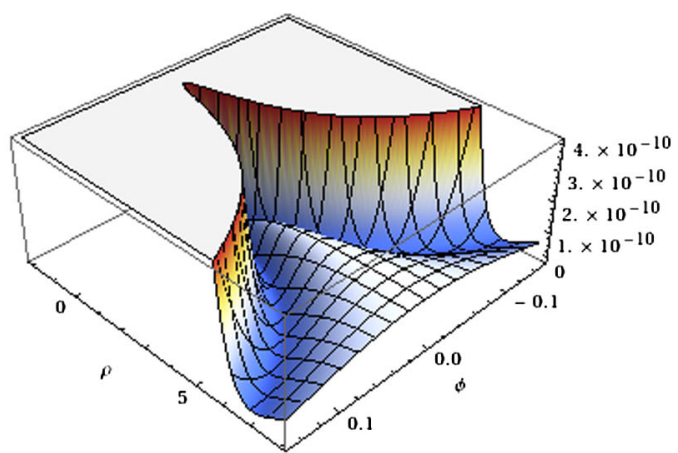

(b2)

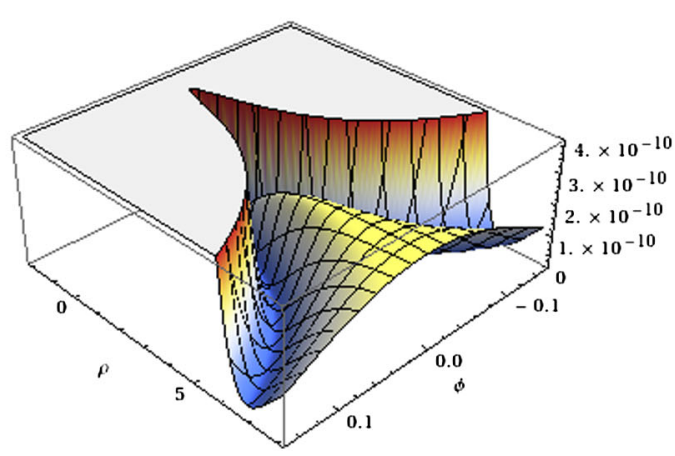

(c2)

FIG. 1. The potential $\hat{W}(\phi, \rho)$ Eq. (42) with values in Planck units and subtraction scale $\mu=M_{p}$. Plot $(a 1): \alpha=4.2 \times 10^{8}, \xi=0$ and $\lambda=0$ (the "usual" Starobinsky model). Next consider $\lambda=0.06$ and: (a2): $\alpha=4.2 \times 10^{8}, \xi=0$, so increasing $\lambda$ lifts up the valley at $\rho=0 .(b 1): \alpha=4.2 \times 10^{8}, \xi=3000,(b 2): \alpha=4.2 \times 10^{8}, \xi=10000 ;(c 1): \alpha=2.2 \times 10^{8}, \xi=3000 ;(c 2): \alpha=2.2 \times 10^{8}, \xi=10000$. Increasing $\xi$ for $\alpha$ fixed [e.g., in plots (b)] brings deeper valleys and compensates for the opposite effect of $\lambda$, while keeping the height of $\hat{W}$ fixed; $\xi$ also controls the position of the valley, relative to plot (a1). Decreasing $\alpha$, for $\xi$ fixed [plots (c) versus (b)], increases the height of $\hat{W}$. Finally, increasing $\lambda$ to $\lambda=0.6$ in the plots above [other than (a1)] simply makes their valleys shallower, without changing the rest. The values of the potential are color encoded with blue (red) for the lowest (highest) value, respectively. 

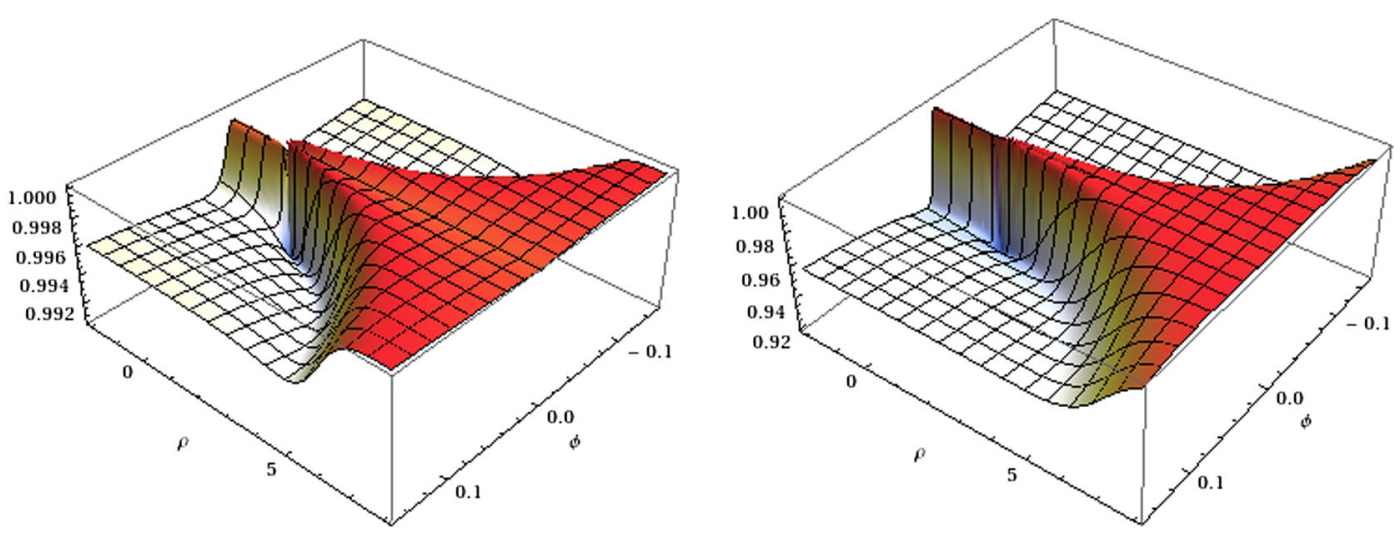

FIG. 2. The relevance of the quantum corrections to the potential in terms of the field values in Planck units. The plots show the twoloop corrected potential $\hat{W}$ of Eq. (42) normalized to its classical value Eq. (10), for various field values, with $\xi=3000, \alpha=4.2 \times 10^{8}$, and $\lambda=0.06$ (left) and $\lambda=0.6$ (right). These plots show that the relative difference quantum effects bring to the potential is small, up to $\varepsilon=1 \%$ reduction for $\lambda=0.06, \varepsilon=6 \%$ reduction for $\lambda=0.6$ or up to $\varepsilon=10 \%$ for $\lambda=1$ (not shown); [note our normalization for $\lambda$, Eq. (1)].

dependence of the quantum corrections). As a result, the classical level analysis in [28] of the Starobinsky-Higgs model does not change significantly at the quantum level, for the generic values of the parameters used here.

With $\hat{W}$ mildly reduced relative to its classical value, by one- and two-loop corrections, then $\eta_{\|}$increases by a similar relative amount (denoted $\varepsilon$ ); see Eqs. (44)-(46) (we ignore here the subleading two-loop wave function correction). Accordingly, one has $\varepsilon \approx 1 \%$ for $\lambda=0.06$, and $\varepsilon \approx 6 \%$ for $\lambda=0.6$. Therefore, the spectral index $n_{s}=$ $1+2 \eta_{\|}-4 \epsilon$ [28] decreases by $2 \varepsilon\left|\eta_{\|}\right|$. For a conservative $\left|\eta_{\|}\right| \sim 0.05$, the decrease of $n_{s}$ is then between 0.002 and 0.006 (depending on $\varepsilon$ ), which is situated between $1 \sigma$ and $3 \sigma$ deviation of $n_{s}$ [59] (ignoring a subleading $\epsilon$ correction relative to $\eta_{\|}$). For the tensor-to-scalar ratio $r=16 \epsilon c_{s}$ [28] a similar calculation gives a relative increase of $2.5 \varepsilon$ from its classical value, or $2.5 \%$ (13\%) for $\lambda=0.06(\lambda=0.6)$, respectively $\left(n_{s}\right.$ and $r$ variations increase with $\lambda$ ).

\section{CONCLUSIONS}

In this work, we analyzed the two-loop matter corrections (of Higgs-like $\phi$ ) to the action in curved spacetime, in the presence of nonminimal coupling $\xi \phi^{2} R$ and of terms quadratic in the curvature scalar $(R)$ and tensors $\left(R_{\mu \nu}\right.$, $\left.R_{\mu \nu \rho \sigma}\right)$. The motivation was to explore the effects of the quantum corrections in classical models of inflation, such as Starobinsky-Higgs inflation. The scalar potential is then an expansion $V(\phi)=V_{0}+V_{1}+V_{2}+\cdots$, where $V_{0}$ is the flat spacetime potential, $V_{1}$ is linear in $R, V_{2}$ contains is quadratic in $R, R_{\mu \nu}, R_{\mu \nu \rho \sigma}$, etc., with coefficients functions of $\phi$. The quantum corrected $V(\phi)$ was computed from the corresponding Callan-Symanzik equations for $V_{0,1,2}$ at the two-loop level. The quantum potential is useful for precision data constraints on the Starobinsky-Higgs model.
While an $R^{2}$ term may be absent classically, it is nevertheless present at the quantum level with a $\phi$-dependent coefficient. Given the presence of this term, the action automatically includes a Starobinsky-like model as a limiting case. Correspondingly, one has a new scalar field (scalaron $\rho$ ) induced geometrically by the $R^{2}$ term. As a result, in the presence of nonminimal coupling multifield inflation is a quantum consequence.

Depending on the field and parameters values one can have dominant Higgs, dominant Starobinsky inflation, or a combination of these, thus giving a unified (quantum) model of Starobinsky-Higgs inflation. In the cases explored, the quantum corrections (sum of one- and two-loop) to the potential are in the region of few percent and increase with the Higgs self-coupling $\lambda$; their impact on the spectral index $n_{s}$ is between $1 \sigma$ and $3 \sigma$ variations (depending on exact $\lambda$ ). Thus, the Starobinsky-Higgs model of inflation is rather stable in the presence of quantum corrections, for generic $\lambda$.

It is known that for a large nonminimal coupling $\xi$, one can integrate the Higgs field in the classical action to recover the "usual" Starobinsky model with a modified scalaron mass that depends on all parameters: $\alpha, \xi, \lambda$. At the quantum level, we showed that integrating $\phi$ generates a "refined" Starobinskylike action with additional terms $\xi^{2} R^{2} \ln ^{n}\left(\xi|R| / \lambda \mu^{2}\right), n=1$, 2 , with implications for inflation (spectral index and scalar-totensor ratio) discussed recently. These terms bring corrections to the scalaron potential $\propto \lambda \rho / M_{p}$ (Einstein frame) and a "running" scalaron mass. Terms like $C^{2} \ln \left(\xi|R| / \mu^{2}\right)$ and $G \ln \left(\xi|R| / \mu^{2}\right)$ were also generated when integrating matter field(s) $(\phi)$ at the loop level.

Finally, in the case when the $R^{2}$ term is absent at the classical level, we stress that the "usual" Starobinsky inflation can still take place. Quantum corrections generate a leading term $\xi^{2} R^{2} \ln \left(M_{p}^{2} / \phi^{2}\right)$, with higher orders 
suppressed by small $\lambda$. For suitable $\xi$ and a small (fixed) Higgs field $\phi^{2} \ll M_{p}^{2} / \xi$, the "usual" Starobinsky inflation is therefore generated at the quantum level alone, which is an interesting result. In this case the Higgs field plays no role other than having a suitable (large) nonminimal coupling to $R$.

\section{ACKNOWLEDGMENTS}

This work was supported by the National Programme Project No. PN/18090101/2018 of the Ministry of Research and Innovation. The author thanks Hyun Min Lee (Seoul, Chung-Ang University) and Andrei Micu (IFIN Bucharest) for interesting discussions on quantum corrections in twofield inflation models.

\section{APPENDIX A: DETAILS ON TWO-LOOP POTENTIAL}

(i) The two-loop potential is found from the CallanSymanzik general equation,

$$
\begin{gathered}
\left(\mu \frac{\partial}{\partial \mu}-\gamma \phi \frac{\partial}{\partial \phi}+\beta_{\lambda} \frac{\partial}{\partial \lambda}+\beta_{\xi} \frac{\partial}{\partial \xi}+\beta_{\Lambda} \frac{\partial}{\partial \Lambda}\right. \\
\left.+\beta_{\kappa_{0}} \frac{\partial}{\partial \kappa_{0}}+\sum_{j=1}^{3} \beta_{\alpha_{j}} \frac{\partial}{\partial \alpha_{j}}\right) V=0,
\end{gathered}
$$

using an expansion in curvature as mentioned in the text; see also Eqs. (1) and (17):

$$
V=V_{0}+V_{1}+V_{2} .
$$

For our massless case, $\beta_{\Lambda}=\beta_{\kappa_{0}}=0 \quad\left(\kappa_{0}\right.$ is the coefficient of $R$ ). The metric is not quantized. $V$ depends on $\alpha_{i}$ only via classical action (1). Then,

$$
\begin{aligned}
& \left(\mu \frac{\partial}{\partial \mu}+\beta_{\lambda} \frac{\partial}{\partial \lambda}+\beta_{\xi} \frac{\partial}{\partial \xi}-\gamma \phi \frac{\partial}{\partial \phi}\right) V \\
& \quad=\beta_{\alpha_{1}} R^{\mu \nu \rho \sigma} R_{\mu \nu \rho \sigma}+\beta_{\alpha_{2}} R^{\mu \nu} R_{\mu \nu}+\beta_{\alpha_{3}} R^{2},
\end{aligned}
$$

where

$$
\begin{aligned}
\beta_{X} & =\frac{d X}{d \ln \mu}, \quad X: \lambda, \xi, \alpha_{j} ; \\
\gamma & =-\frac{d \ln \phi}{d \ln \mu}=\frac{d \ln Z_{\phi}^{1 / 2}}{d \ln \mu} ; \quad \phi_{B}=Z_{\phi}^{1 / 2} \phi \mu^{-\epsilon} .
\end{aligned}
$$

Equating terms of similar curvature in (A3) gives the CS equations for $V_{0}, V_{1}, V_{2}$. For $V_{0}$

$$
\left(\mu \frac{\partial}{\partial \mu}+\beta_{\lambda} \frac{\partial}{\partial \lambda}-\gamma \phi \frac{\partial}{\partial \phi}\right) V_{0}=0,\left.\quad V_{0}^{(4)}\right|_{\phi=\mu}=\lambda,
$$

where $V_{0}^{(4)}=d^{4} V_{0} / d \phi^{4}$. To solve it, first differentiate it four times with respect to $\phi$ :

$$
\left(\mu \frac{\partial}{\partial \mu}+\beta_{\lambda} \frac{\partial}{\partial \lambda}-4 \gamma-\gamma \phi \frac{\partial}{\partial \phi}\right) V_{0}^{(4)}=0 .
$$

Since $V_{0}^{(4)}$ is dimensionless, it must depend on $\mu$ and $\phi$ only via their ratio. Therefore,

$$
\begin{aligned}
\left(\frac{\partial}{\partial t}+\tilde{\beta}_{\lambda} \frac{\partial}{\partial \lambda}-4 \tilde{\gamma}\right) V_{0}^{(4)} & =0, \\
t=\ln (\mu / \phi),\left.\quad V_{0}^{(4)}\right|_{t=0} & =\lambda,
\end{aligned}
$$

where

$$
\begin{aligned}
& \tilde{\beta}_{\lambda}=\frac{\beta_{\lambda}}{1+\gamma}=\beta_{\lambda}^{(1)}+\beta_{\lambda}^{(2)}+\mathcal{O}\left(\lambda^{4}\right) \\
& \tilde{\gamma}=\frac{\gamma}{1+\gamma}=\gamma^{(2)}+\mathcal{O}\left(\lambda^{4}\right) .
\end{aligned}
$$

The superscripts on $\beta_{\lambda}^{(k)}$ and $\gamma^{(k)}$ denote the $k$ th-loop order correction: $\beta_{\lambda}^{(1)} \sim \lambda^{2}, \beta_{\lambda}^{(2)} \sim \lambda^{3}, \gamma^{(2)} \sim \lambda^{2}$, etc. Writing $V_{0}^{(4)}$ as an expansion in powers of $\lambda$ up to three-loop corrections,

$$
\begin{aligned}
& V_{0}^{(4)}=\mathcal{V}^{(0)}+\mathcal{V}^{(1)}+\mathcal{V}^{(2)}+\mathcal{O}\left(\lambda^{4}\right), \\
& \mathcal{V}^{(j)} \sim \lambda^{j+1}, \quad j=0,1,2 .
\end{aligned}
$$

then $\mathcal{V}^{(0)}=\lambda$. Then in order $\lambda^{2}$ the CS equation gives

$$
\frac{\partial \mathcal{V}^{(1)}}{\partial t}+\beta_{\lambda}^{(1)} \frac{\partial \mathcal{V}^{(0)}}{\partial \lambda}=\mathcal{O}\left(\lambda^{3}\right),
$$

with a solution $\mathcal{V}^{(1)}=-\beta_{\lambda}^{(1)} t+$ constant. Next,

$\frac{\partial \mathcal{V}^{(2)}}{\partial t}+\beta_{\lambda}^{(2)} \frac{\partial \mathcal{V}^{(0)}}{\partial \lambda}+\beta_{\lambda}^{(1)} \frac{\partial \mathcal{V}^{(1)}}{\partial \lambda}-4 \gamma^{(2)} \mathcal{V}^{(0)}=\mathcal{O}\left(\lambda^{4}\right)$,

which is integrated with respect to $t$. We find

$$
V_{0}^{(4)}=\lambda-\beta_{\lambda}^{(1)} t+\left(4 \gamma^{(2)} \lambda-\beta_{\lambda}^{(2)}\right) t+\beta_{\lambda}^{(1)} \frac{\partial \beta_{\lambda}^{(1)}}{\partial \lambda} \frac{t^{2}}{2} .
$$

In (A12), we denote by $u_{1}$ and $u_{2}$ the coefficients of $t$ and $t^{2}$, then integrate (A12) over $\phi$ four times, with $t$ as in (A7), then 


$$
\begin{aligned}
V_{0}= & {\left[\frac{\lambda}{4 !}+\frac{25}{288} u_{1}+\frac{415}{1728} u_{2}\right] \phi^{4} } \\
& -\frac{1}{288}\left(6 u_{1}+25 u_{2}\right) \phi^{4} \ln \frac{\phi^{2}}{\mu^{2}}+\frac{u_{2}}{96} \phi^{4} \ln ^{2} \frac{\phi^{2}}{\mu^{2}} \\
= & \frac{\lambda}{4 !} \phi^{4}+\frac{\lambda^{2}}{16 \kappa} \phi^{4}\left[\ln \frac{\phi^{2}}{\mu^{2}}-\frac{25}{6}\right] \\
& +\frac{\lambda^{3}}{32 \kappa^{2}} \phi^{4}\left[\frac{515}{6}-29 \ln \frac{\phi^{2}}{\mu^{2}}+3 \ln ^{2} \frac{\phi^{2}}{\mu^{2}}\right],
\end{aligned}
$$

as quoted in the text; in the last step, we use (Appendix B):

$$
\beta_{\lambda}^{(1)}=\frac{3 \lambda^{2}}{\kappa}, \quad \beta_{\lambda}^{(2)}=-\frac{17}{3} \frac{\lambda^{3}}{\kappa^{2}}, \quad \gamma^{(2)}=\frac{\lambda^{2}}{12 \kappa^{2}} .
$$

(ii) Further, the CS equation for $V_{1}$ is found from (A3)

$$
\left(\mu \frac{\partial}{\partial \mu}+\beta_{\lambda} \frac{\partial}{\partial \lambda}+\beta_{\xi} \frac{\partial}{\partial \xi}-\gamma \phi \frac{\partial}{\partial \phi}\right) V_{1}=0 .
$$

with

$$
V_{1}^{(2)}=\frac{\partial^{2} V_{1}}{\partial \phi^{2}},\left.\quad V_{1}^{(2)}\right|_{\phi=\mu}=\xi R
$$

After applying $\partial^{2} / \partial \phi^{2}$, one has

$$
\begin{aligned}
\left(\frac{\partial}{\partial t}+\tilde{\beta}_{\lambda} \frac{\partial}{\partial \lambda}+\tilde{\beta}_{\xi} \frac{\partial}{\partial \xi}-2 \tilde{\gamma}\right) V_{1}^{(2)} & =0, \\
\left.V_{1}^{(2)}\right|_{t=0} & =\xi R,
\end{aligned}
$$

with

$$
\begin{aligned}
\tilde{\beta}_{\xi} & =\frac{\beta_{\xi}}{1+\gamma}=\beta_{\xi}^{(1)}+\beta_{\xi}^{(2)}-\gamma^{(2)} \beta_{\xi}^{(1)}+\mathcal{O}\left(\lambda^{4}\right), \\
\beta_{\xi}^{(j)} & \sim \lambda^{j}
\end{aligned}
$$

The solution has a loop expansion

$$
\begin{aligned}
V_{1}^{(2)} & =W^{(0)}+W^{(1)}+W^{(2)}+\mathcal{O}\left(\lambda^{3}\right), \\
W^{(j)} & \sim \lambda^{j}, \quad j=0,1,2,3 .
\end{aligned}
$$

Then $W^{(0)}=\xi R$. The CS equation then splits into two equations for the remaining $W^{(j)}$

$$
\begin{aligned}
& \frac{\partial W^{(1)}}{\partial t}+\beta_{\xi}^{(1)} \frac{\partial W^{(0)}}{\partial \xi}=\mathcal{O}\left(\lambda^{2}\right) \\
& \frac{\partial W^{(2)}}{\partial t}+\beta_{\lambda}^{(1)} \frac{\partial W^{(1)}}{\partial \lambda}+\beta_{\xi}^{(1)} \frac{\partial W^{(1)}}{\partial \xi}+\beta_{\xi}^{(2)} \frac{\partial W^{(0)}}{\partial \xi} \\
& \quad-2 \gamma^{(2)} W^{(0)}=\mathcal{O}\left(\lambda^{3}\right)
\end{aligned}
$$

Then $W^{(1)}=-\beta_{\xi}^{(1)} R t$ which is used to find $W^{(2)}$. Adding these together, then

$$
\begin{array}{rlr}
V_{1}^{(2)}= & R\left[\xi-\beta_{\xi}^{(1)} t+\left(\beta_{\lambda}^{(1)} \frac{\partial}{\partial \lambda} \beta_{\xi}^{(1)}+\beta_{\xi}^{(1)} \frac{\partial}{\partial \xi} \beta_{\xi}^{(1)}\right) \frac{t^{2}}{2}\right. \\
& \left.+\left(2 \gamma^{(2)} \xi-\beta_{\xi}^{(2)}\right) t\right] .
\end{array}
$$

In (A21), we denote by $z_{1}, z_{2}$ the coefficients of $t$ and $t^{2}$ and integrate twice over $\phi$, then

$$
\begin{aligned}
V_{1} & =R \phi^{2}\left\{\frac{\xi}{2}+\frac{3}{4} z_{1}+\frac{7}{4} z_{2}-\frac{1}{4}\left(z_{1}+3 z_{2}\right) \ln \frac{\phi^{2}}{\mu^{2}}+\frac{1}{8} z_{2} \ln ^{2} \frac{\phi^{2}}{\mu^{2}}\right\} \\
& =R \phi^{2}\left\{\frac{\xi}{2}+\frac{\lambda}{4 \kappa}\left(\xi+\frac{1}{6}\right)\left(\ln \frac{\phi^{2}}{\mu^{2}}-3\right)-\frac{\lambda^{2}}{4 \kappa^{2}}\left(\frac{7}{6} \xi+\frac{13}{36}\right)\left(\ln \frac{\phi^{2}}{\mu^{2}}-3\right)+\frac{2 \lambda^{2}}{\kappa^{2}} \frac{1}{4}\left(\xi+\frac{1}{6}\right)\left(7-3 \ln \frac{\phi^{2}}{\mu^{2}}+\frac{1}{2} \ln ^{2} \frac{\phi^{2}}{\mu^{2}}\right)\right\},
\end{aligned}
$$

where in the last step we replaced the beta functions (see Appendix B)

$\beta_{\xi}^{(1)}=\frac{\lambda}{\kappa}\left(\xi+\frac{1}{6}\right), \quad \beta_{\xi}^{(2)}=-\frac{\lambda^{2}}{\kappa^{2}}\left(\xi+\frac{1}{6}\right)-\frac{\lambda^{2}}{\kappa^{2}} \frac{7}{36}$

$(\xi=-1 / 6$ is conformal at one-loop only). (iii) Finally, compute the two-loop corrections to $V_{2}$

$$
V_{2}=-\tilde{\alpha}_{1} R_{\mu \nu \rho \sigma} R^{\mu \nu \rho \sigma}-\tilde{\alpha}_{2} R_{\mu \nu} R^{\mu \nu}-\tilde{\alpha}_{3} R^{2}
$$

The CS equation is

$$
\left(\mu \frac{\partial}{\partial \mu}+\beta_{\lambda} \frac{\partial}{\partial \lambda}+\beta_{\xi} \frac{\partial}{\partial \xi}-\gamma \phi \frac{\partial}{\partial \phi}\right)\left(-\tilde{\alpha}_{i}\right)=\beta_{\alpha_{i}},
$$$$
i=1,2,3 \text {. }
$$ 
giving

$$
\left(\frac{\partial}{\partial t}+\tilde{\beta}_{\lambda} \frac{\partial}{\partial \lambda}+\tilde{\beta}_{\xi} \frac{\partial}{\partial \xi}\right)\left(-\tilde{\alpha}_{i}\right)=\tilde{\beta}_{\alpha_{i}},\left.\quad \tilde{\alpha}_{i}\right|_{t=0}=\alpha_{i} .
$$

Here $\tilde{\beta}_{\lambda}$ and $\tilde{\beta}_{\xi}$ are given in Eqs. (A8) and (A18) and $\tilde{\beta}_{\alpha_{i}}=\frac{\beta_{\alpha_{i}}}{1+\gamma}=\beta_{\alpha_{i}}\left(1-\gamma^{(2)}-\gamma^{(3)}\right)+\mathcal{O}\left(\lambda^{4}\right)$,

with $\beta_{\alpha_{i}}=d \alpha_{i} / d(\ln \mu)$. A solution is expanded in powers of $\lambda$,

$$
\tilde{\alpha}_{i}=\alpha_{i}^{(0)}+\alpha_{i}^{(1)}+\cdots, \quad \alpha_{i}^{(k)} \sim \lambda^{k},
$$

giving

$-\frac{\partial \alpha_{i}^{(0)}}{\partial t}=\beta_{\alpha_{i}}^{(1)}, \quad-\frac{\partial \alpha_{i}^{(1)}}{\partial t}-\beta_{\xi}^{(1)} \frac{\partial \alpha_{i}^{(0)}}{\partial \xi}=\beta_{\alpha_{i}}^{(2)}$.

Here $\beta_{\alpha_{i}}^{(1)} \sim \lambda^{0}$ and $\beta_{\alpha_{i}}^{(2)} \sim \lambda$ are one- and two-loop corrections to $\beta_{\alpha_{i}}$. Then at two-loop

$$
-\tilde{\alpha}_{i}=-\alpha_{i}+\beta_{\alpha_{i}}^{(1)} t-\beta_{\xi}^{(1)} \frac{\partial \beta_{\alpha_{i}}^{(1)}}{\partial \xi} \frac{t^{2}}{2}+\beta_{\alpha_{i}}^{(2)} t .
$$

giving, with $t=\ln \mu / \phi$ :

$$
\begin{aligned}
-\tilde{\alpha}_{1}= & -\alpha_{1}+\left(\frac{-1}{180 \kappa}\right) \ln \frac{\mu}{\phi} \\
-\tilde{\alpha}_{2}= & -\alpha_{2}+\left(\frac{1}{180 \kappa}\right) \ln \frac{\mu}{\phi} \\
-\tilde{\alpha}_{3}= & -\alpha_{3}-\frac{1}{2 \kappa}\left(\xi+\frac{1}{6}\right)^{2} \ln \frac{\mu}{\phi} \\
& +\frac{\lambda}{2 \kappa^{2}}\left(\xi+\frac{1}{6}\right)^{2} \ln ^{2} \frac{\mu}{\phi},
\end{aligned}
$$

quoted in the text. Above we used (Appendix B)

$$
\begin{aligned}
& \beta_{\alpha_{1}}^{(1)}=-\frac{1}{180 \kappa}, \quad \beta_{\alpha_{2}}^{(1)}=\frac{1}{180 \kappa}, \\
& \beta_{\alpha_{3}}^{(1)}=-\frac{1}{2 \kappa}\left(\xi+\frac{1}{6}\right)^{2}, \quad \beta_{\alpha_{1}}^{(2)}=\beta_{\alpha_{2}}^{(2)}=\beta_{\alpha_{3}}^{(2)}=0 .
\end{aligned}
$$

\section{APPENDIX B: TWO-LOOP BETA FUNCTIONS}

Here we provide the derivation of the beta functions $\beta_{\xi}$ and $\beta_{\alpha_{j}}$ used in the previous section. With initial $L$ as in (1), these are found from the two-loop counterterms $\delta L$ to $L[41] \delta L$ is of the same form as the terms in initial $L$ Eq. (1):

$$
\begin{aligned}
\frac{\delta L}{\sqrt{\tilde{g}}}= & -\frac{1}{2} \delta Z_{\phi} \phi \square \phi-\frac{1}{2} \delta \xi R \phi^{2}-\frac{1}{4 !} \delta \lambda \phi^{4} \\
& +\delta \alpha_{1} R_{\mu \nu \rho \sigma} R^{\mu \nu \rho \sigma}+\delta \alpha_{2} R_{\mu \nu} R^{\mu \nu}+\delta \alpha_{3} R^{2}
\end{aligned}
$$

(a) First, $\delta Z_{\phi}=Z_{\phi}-1$ and

$$
\phi_{B}=\mu^{-\epsilon} Z_{\phi}^{1 / 2} \phi
$$

with

$Z_{\phi}=1-\frac{\lambda^{2}}{24 \kappa^{2} \epsilon}, \quad \gamma_{\phi}^{(2)}=\frac{d \ln Z_{\phi}^{1 / 2}}{d \ln \mu}=\frac{\lambda^{2}}{12 \kappa^{2}}$.

where we used $\beta_{\lambda}$ derived below:

$$
\begin{aligned}
& \lambda_{B}=\mu^{2 \epsilon}(\lambda+\delta \lambda) Z_{\phi}^{-2}, \\
& \delta \lambda \equiv \lambda\left(Z_{\lambda}-1\right)=\delta \lambda^{(1)}+\delta \lambda^{(2)}
\end{aligned}
$$

with

$\delta \lambda^{(1)}=\frac{-3 \lambda^{2}}{(-2 \epsilon) \kappa}, \quad \delta \lambda^{(2)}=\frac{\lambda^{3}}{\kappa^{2}}\left(\frac{9}{4 \epsilon^{2}}-\frac{3}{2 \epsilon}\right)$

in the MS scheme. Since $\frac{d \lambda_{B}}{d \ln \mu}=0$, with $Z_{\phi}$ above and $\beta_{\lambda}=d \lambda / d(\ln \mu)$, then at two-loop

$$
\beta_{\lambda}=\beta_{\lambda}^{(1)}+\beta_{\lambda}^{(2)}=-2 \epsilon \lambda+\frac{3 \lambda^{2}}{\kappa}-\frac{17}{3} \frac{\lambda^{3}}{\kappa^{2}}
$$

(b) Further, we have:

$$
\xi_{B}=(\xi+\delta \xi) Z_{\phi}^{-1}, \quad \delta \xi \equiv \xi\left(Z_{\xi}-1\right)=\delta \xi^{(1)}+\delta \xi^{(2)}
$$

with [41]

$$
\begin{aligned}
& \delta \xi^{(1)}=\frac{-\lambda}{\kappa(-2 \epsilon)}\left(\xi+\frac{1}{6}\right) \\
& \delta \xi^{(2)}=\frac{\lambda^{2}}{2 \kappa^{2} \epsilon^{2}}\left(\xi+\frac{1}{6}\right)-\frac{\lambda^{2}}{4 \kappa^{2} \epsilon}\left(\xi+\frac{7}{36}\right)
\end{aligned}
$$

Together with $Z_{\phi}$ and $\xi_{B}=\xi Z_{\xi} Z_{\phi}^{-1}$, (B8) gives the two-loop corrected $\beta_{\xi}=d \xi / d(\ln \mu)$ as

$\beta_{\xi}=\beta_{\xi}^{(1)}+\beta_{\xi}^{(2)}=\frac{\lambda}{\kappa}\left(\xi+\frac{1}{6}\right)+\frac{-\lambda^{2}}{\kappa^{2}}\left(\xi+\frac{1}{6}\right)-\frac{7}{36} \frac{\lambda^{2}}{\kappa^{2}}$. 
(c) Finally, one has

$$
\begin{aligned}
& \alpha_{j B}=\mu^{-2 \epsilon}\left(\alpha_{j}+\delta \alpha_{j}\right), \\
& \delta \alpha_{j} \equiv \alpha_{j}\left(Z_{\alpha_{j}}-1\right)=\delta \alpha_{j}^{(1)}+\delta \alpha_{j}^{(2)}, \quad j: 1,2,3 .
\end{aligned}
$$

with [41]

$\delta \alpha_{1}^{(1)}=\frac{1}{(-2 \epsilon) \kappa} \frac{1}{180}=-\delta \alpha_{2}^{(1)}, \quad \delta \alpha_{1}^{(2)}=\delta \alpha_{2}^{(2)}=0$,

and

$$
\begin{aligned}
& \delta \alpha_{3}^{(1)}=\frac{1}{2(-2 \epsilon) \kappa}\left(\xi+\frac{1}{6}\right)^{2} \\
& \delta \alpha_{3}^{(2)}=-\frac{\lambda}{2 \kappa^{2}(2 \epsilon)^{2}}\left(\xi+\frac{1}{6}\right)^{2}
\end{aligned}
$$

With $\beta_{\alpha_{j}}=d \alpha_{j} / d(\ln \mu)$ from $d \alpha_{j B} / d \ln \mu=0$ and $\alpha_{j B}=\mu^{-2 \epsilon} \alpha_{j} Z_{\alpha_{j}}$, one finds

$$
\beta_{\alpha_{j}}=\beta_{\alpha_{j}}^{(1)}+\beta_{\alpha_{j}}^{(2)}
$$

where

$$
\begin{aligned}
& \beta_{\alpha_{1}}^{(1)}=2 \epsilon \alpha_{1}-\frac{1}{180 \kappa}, \\
& \beta_{\alpha_{2}}^{(1)}=2 \epsilon \alpha_{2}+\frac{1}{180 \kappa}, \\
& \beta_{\alpha_{3}}^{(1)}=2 \epsilon \alpha_{3}-\frac{1}{2 \kappa}\left(\xi+\frac{1}{6}\right)^{2},
\end{aligned}
$$

while $\beta_{\alpha_{1,2,3}}^{(2)}=0$ (as expected).
[1] A. H. Guth, The inflationary universe: A possible solution to the horizon and flatness problems, Phys. Rev. D 23, 347 (1981).

[2] A. A. Starobinsky, A new type of isotropic cosmological models without singularity, Phys. Lett. 91B, 99 (1980); Spectrum of relict gravitational radiation and the early state of the universe, Pis'ma Zh. Eksp. Teor. Fiz. 30, 719 (1979) [JETP Lett. 30, 682 (1979)].

[3] K. Sato, First order phase transition of a vacuum and expansion of the universe, Mon. Not. R. Astron. Soc. 195, 467 (1981).

[4] A. D. Linde, A new inflationary universe scenario: A possible solution of the horizon, flatness, homogeneity, isotropy and primordial monopole problems, Phys. Lett. 108B, 389 (1982).

[5] A. Albrecht and P. J. Steinhardt, Cosmology for Grand Unified Theories with Radiatively Induced Symmetry Breaking, Phys. Rev. Lett. 48, 1220 (1982).

[6] A. D. Linde, Chaotic inflation, Phys. Lett. 129B, 177 (1983).

[7] S. W. Hawking and I. G. Moss, Supercooled phase transitions in the very early universe, Phys. Lett. 110B, 35 (1982).

[8] J. B. Hartle and S. W. Hawking, Wave function of the universe, Phys. Rev. D 28, 2960 (1983).

[9] For a review K. Sato and J. Yokoyama, Inflationary cosmology: First 30+ years, Int. J. Mod. Phys. D 24, 1530025 (2015).

[10] P. A. R. Ade et al. (Planck Collaboration), Planck 2015 results. XX. Constraints on inflation, Astron. Astrophys. 594, A20 (2016).
[11] J. L. Cervantes-Cota and H. Dehnen, Induced gravity inflation in the standard model of particle physics, Nucl. Phys. B442, 391 (1995).

[12] F. L. Bezrukov and M. Shaposhnikov, The Standard Model Higgs boson as the inflaton, Phys. Lett. B 659, 703 (2008).

[13] A. O. Barvinsky, A. Y. Kamenshchik, and A. A. Starobinsky, Inflation scenario via the Standard Model Higgs boson and LHC, J. Cosmol. Astropart. Phys. 11 (2008) 021.

[14] F. Bezrukov, A. Magnin, M. Shaposhnikov, and S. Sibiryakov, Higgs inflation: Consistency and generalisations, J. High Energy Phys. 01 (2011) 016.

[15] O. Lebedev and H. M. Lee, Higgs portal inflation, Eur. Phys. J. C 71, 1821 (2011).

[16] D. I. Kaiser, Nonminimal Couplings in the Early Universe: Multifield Models of Inflation and the Latest Observations, edited by T. Asselmeyer-Maluga, At the Frontier of Spacetime. Fundamental Theories of Physics Vol. 183 (Springer, Cham, 2016).

[17] F. L. Bezrukov, A. Magnin, and M. Shaposhnikov, Standard Model Higgs boson mass from inflation, Phys. Lett. B 675, 88 (2009).

[18] F. Bezrukov and M. Shaposhnikov, Standard Model Higgs boson mass from inflation: Two loop analysis, J. High Energy Phys. 07 (2009) 089.

[19] Y. Ema, Higgs scalaron mixed inflation, Phys. Lett. B 770, 403 (2017).

[20] Y. Hamada, H. Kawai, K. y. Oda, and S. C. Park, Higgs inflation from Standard Model criticality, Phys. Rev. D 91, 053008 (2015).

[21] M. Atkins and X. Calmet, Remarks on Higgs inflation, Phys. Lett. B 697, 37 (2011). 
[22] G. F. Giudice and H. M. Lee, Unitarizing Higgs inflation, Phys. Lett. B 694, 294 (2011).

[23] F. Bezrukov, A. Magnin, M. Shaposhnikov, and S. Sibiryakov, Higgs inflation: Consistency and generalisations, J. High Energy Phys. 01 (2011) 016.

[24] J. L. F. Barbon and J. R. Espinosa, On the naturalness of Higgs inflation, Phys. Rev. D 79, 081302 (2009).

[25] T. Inagaki, R. Nakanishi, and S. D. Odintsov, Non-minimal two-loop inflation, Phys. Lett. B 745, 105 (2015).

[26] X. Calmet, I. Kuntz, and I. G. Moss, Non-minimal coupling of the Higgs boson to curvature in an inflationary universe, Found. Phys. 48, 110 (2018).

[27] L. Alvarez-Gaume, A. Kehagias, C. Kounnas, D. Lüst, and A. Riotto, Aspects of quadratic gravity, Fortschr. Phys. 64, 176 (2016).

[28] M. He, A. A. Starobinsky, and J. Yokoyama, Inflation in the mixed Higgs- $R^{2}$ model, arXiv:1804.00409.

[29] S. Kaneda and S. V. Ketov, Starobinsky-like two-field inflation, Eur. Phys. J. C 76, 26 (2016).

[30] X. Calmet and I. Kuntz, Higgs Starobinsky inflation, Eur. Phys. J. C 76, 289 (2016).

[31] G. F. Giudice and H. M. Lee, Starobinsky-like inflation from induced gravity, Phys. Lett. B 733, 58 (2014).

[32] T. d. P. Netto, A. M. Pelinson, I. L. Shapiro, and A. A. Starobinsky, From stable to unstable anomaly-induced inflation, Eur. Phys. J. C 76, 544 (2016).

[33] T. Mori, K. Kohri, and J. White, Multi-field effects in a simple extension of $R^{2}$ inflation, J. Cosmol. Astropart. Phys. 10 (2017) 044.

[34] Y.C. Wang and T. Wang, Primordial perturbations generated by Higgs field and $R^{2}$ operator, Phys. Rev. D 96, 123506 (2017).

[35] D. S. Gorbunov and A. G. Panin, Are $R^{2}$ - and Higgsinflations really unlikely? Phys. Lett. B 743, 79 (2015).

[36] F. L. Bezrukov and D. S. Gorbunov, Distinguishing between $R^{2}$-inflation and Higgs-inflation, Phys. Lett. B 713, 365 (2012).

[37] D. Gorbunov and A. Tokareva, $R^{2}$-inflation with conformal SM Higgs field, J. Cosmol. Astropart. Phys. 12 (2013) 021.

[38] R. Costa and H. Nastase, Conformal inflation from the Higgs, J. High Energy Phys. 06 (2014) 145.

[39] H. Kodama and M. Sasaki, Cosmological perturbation theory, Prog. Theor. Phys. Suppl. 78, 1 (1984).

[40] S. D. Odintsov, Two loop effective potential in quantum field theory in curved space-time, Phys. Lett. B 306, 233 (1993).

[41] L. Parker and D. Toms, Quantum Field Theory in Curved Space-Time-Quantized Fields and Gravity (Cambridge University Press, Cambridge, England, 2009).

[42] E. Elizalde and S. D. Odintsov, Renormalization group improved effective potential for gauge theories in curved space-time, Phys. Lett. B 303, 240 (1993); Coleman-Weinberg approach in distorted space-time, Russ. Phys. J. 37, 25 (1994).

[43] E. Elizalde and S. D. Odintsov, Renormalization group improved effective Lagrangian for interacting theories in curved space-time, Phys. Lett. B 321, 199 (1994).

[44] E. Elizalde and S. D. Odintsov, Renormalization group improved effective potential for interacting theories with several mass scales in curved space-time, Z. Phys. C 64, 699 (1994).

[45] D. Gorbunov and V. Rubakov, Introduction to the Theory of the Early Universe (World Scientific, Singapore, 2011).

[46] A. Kehagias, A. Moradinezhad Dizgah, and A. Riotto, Remarks on the Starobinsky model of inflation and its descendants, Phys. Rev. D 89, 043527 (2014).

[47] A. A. Starobinsky, The perturbation spectrum evolving from a nonsingular, initially de Sitter cosmology, and the microwave background anisotropy, Sov. Astron. Lett. 9, 302 (1983).

[48] S. W. Hawking, T. Hertog, and H. S. Reall, Trace anomaly driven inflation, Phys. Rev. D 63, 083504 (2001).

[49] T. Faulkner, M. Tegmark, E. F. Bunn, and Y. Mao, Constraining $f(R)$ gravity as a scalar tensor theory, Phys. Rev. D 76, 063505 (2007).

[50] Z. Lalak and Ł. Nakonieczny, Darkflation-one scalar to rule them all?, Phys. Dark Universe 15, 125 (2017).

[51] M. Demmel, F. Saueressig, and O. Zanusso, A proper fixed functional for four-dimensional quantum Einstein gravity, J. High Energy Phys. 08 (2015) 113.

[52] L. H. Liu, T. Prokopec, and A. A. Starobinsky, Inflation in an effective gravitational model and asymptotic safety, Phys. Rev. D 98, 043505 (2018).

[53] S. Lee, Modified Starobinsky inflation, arXiv:1804.01678.

[54] R. Myrzakulov, S. Odintsov, and L. Sebastiani, Inflationary universe from higher-derivative quantum gravity, Phys. Rev. D 91, 083529 (2015).

[55] R. Myrzakulov, S. Odintsov, and L. Sebastiani, Inflationary universe from higher derivative quantum gravity coupled with scalar electrodynamics, Nucl. Phys. B907, 646 (2016).

[56] E. Elizalde, S. D. Odintsov, L. Sebastiani, and R. Myrzakulov, Beyond-one-loop quantum gravity action yielding both inflation and late-time acceleration, Nucl. Phys. B921, 411 (2017).

[57] L. Sebastiani and R. Myrzakulov, $F(R)$ gravity and inflation, Int. J. Geom. Methods Mod. Phys. 12, 1530003 (2015).

[58] Z. Lalak, D. Langlois, S. Pokorski, and K. Turzynski, Curvature and isocurvature perturbations in two-field inflation, J. Cosmol. Astropart. Phys. 07 (2007) 014.

[59] Y. Akrami et al. (Planck Collaboration), Planck 2018 results. X. Constraints on inflation, arXiv:1807.06211. 